\title{
Cecal Hemorrhage, CTCAE
}

National Cancer Institute

\section{Source}

National Cancer Institute. Cecal Hemorrhage, CT CAE. NCI Thesaurus. Code C143358.

A disorder characterized by bleeding from the cecum. 